\title{
PREVALÊNCIA DO ALEITAMENTO MATERNO EXCLUSIVO E FATORES DE RISCO
}

\section{Leylla Lays Alves e Silva}

Enfermeira. Especialista em Saúde Pública pelo Instituto de Ensino Superior Múltiplo (IESM). Pós-Graduanda em Enfermagem Obstétrica pela Faculdade do Médio Parnaíba (Famep), Brasil.

\section{Ingred Pereira Cirino}

Enfermeira. Especialista em Enfermagem do trabalho pela Faculdade do Médio Parnaíba (Famep). Mestranda do Programa de Pós-Graduação em Ciências e Saúde pela Universidade Federal do Piauí (UFPI), Brasil.

\section{Marcela de Sousa Santos}

Enfermeira pela Universidade Federal do Piauí (UFPI), Brasil.

\section{Edina Araújo Rodrigues Oliveira}

Enfermeira. Mestre em Enfermagem. Doutoranda em Nutrição em Saúde Pública pela. Universidade de São Paulo (USP). Docente do curso de Bacharelado em Enfermagem da Universidade Federal do Piauí (UFPI), Brasil.

\section{Artemizia Francisca de Sousa}

Nutricionista. Mestre em ciências e Saúde. Doutoranda em Nutrição em Saúde Pública pela Universidade de São Paulo (USP). Docente do curso de Nutrição da Universidade Federal do Piauí (UFPI), Brasil.

\section{Luisa Helena de Oliveira Lima}

Enfermeira. Mestre em Enfermagem. Doutora em Enfermem. Docente do curso de Bacharelado em Enfermagem da Universidade Federal do Piauí (UFPI), Brasil.
RESUMO: Investigar a prevalência do aleitamento materno exclusivo ao nascer e seus fatores de risco. A população foi composta por todas as crianças nascidas vivas e suas respectivas mães no período de janeiro a dezembro de 2015, no alojamento conjunto de um hospital público do Piauí, totalizando 546 nascidos vivos. A pesquisa foi aprovada pelo Comitê de Ética com parecer 1.144.279. Das mães pesquisadas, $97,6 \%$ realizaram pré-natal, destas $67 \%$ receberam orientações sobre aleitamento materno. A prevalência de aleitamento materno exclusivo e aleitamento materno foi de 92,7 e $2,9 \%$, respectivamente. Constatou-se que $20,7 \%$ das crianças faziam uso de chupeta e $4,4 \%$ de mamadeira. Faz-se necessário realizar intervenções de prevenção dos fatores de risco e desmame precoce por meio de atividades educativas que orientem mães e familiares envolvidos na amamentação.

PALAVRAS-CHAVE: Aleitamento materno; Promoção da saúde; Saúde da criança.

\section{PREVALENCE OF EXCLUSIVE BREASTFEEDING AND RISK FACTORS}

ABSTRACT: The prevalence of exclusive breast-feeding at birth and its risk factors are assessed. Population comprised all live births $(n=546)$ and their respective mothers between January and December 2015, in a hostel on the premises of a public hospital in Piauí, Brazil. Research was approved by the Committee for Ethics (protocol n. 1.144.279). Further, $97.6 \%$ of mothers underwent pre-natal and $67 \%$ were counseled for breast-feeding. The prevalence of exclusive breast-feeding and breast-feeding was 92.7 and $2.9 \%$, respectively. Moreover, $20.7 \%$ of the children use pacifiers and $4.4 \%$ the feeding bottle. Interventions for the prevention of risk factors and early weaning are necessary through educational activities that serve as guidelines to mother and family members involved in breast-feeding.

KEY WORDS: Breast feeding; Health promotion; Child Health.

\section{INTRODUÇÃO}

É cientificamente comprovada a superioridade do leite materno sobre outras formas de alimentar uma criança, isto posto, o aleitamento materno (AM) se apresenta como umas das principais ações da atenção primária à saúde por contribuir para a redução da prevalência de doenças e trazer uma série de benefícios tanto para o bebê quanto para a mãe $e^{1}$.

O AM melhora a sobrevivência, a saúde e o desenvolvimento 
de todas as crianças ${ }^{2}$. Este alimento é considerado ideal para o lactente pelas suas propriedades nutritivas e protetoras contra infecções gastrintestinais, respiratórias, urinárias e alergias ${ }^{3}$.

Os efeitos benéficos da amamentação se estendem a todo o ciclo vital, reduzindo o risco e a gravidade de ocorrência de problemas que se manifestam tardiamente, como o grupo complexo das doenças crônicas não transmissíveis, entre outras comorbidades próprias da vida adulta e da senescênciá .

Apesar da superioridade do leite materno sobre outros tipos de leite, ainda é baixa a prevalência do aleitamento materno, configurando importante problema de saúde pública ${ }^{5}$. Na última pesquisa nacional sobre AM foi observado que apenas $41 \%$ dos bebês menores de seis meses são alimentados exclusivamente com leite materno ${ }^{6}$. Esta taxa é semelhante à média mundial que é de $39 \%$, mas encontra-se distante do percentual ideal preconizado pelos órgãos nacionais e internacionais que é entre $90 \%$ e $100 \%{ }^{7}$. Conquanto, o aumento das taxas de amamentação exclusiva tem sido responsável por salvar a vida de cerca de 6 milhões de crianças a cada ano no mundo ${ }^{8}$. Nesta perspectiva, o objetivo desse estudo é investigar a prevalência do aleitamento materno exclusivo (AME) ao nascer e seus fatores de risco.

\section{METODOLOGIA}

Trata-se de um estudo quantitativo, descritivo-exploratório e transversal, realizado no alojamento conjunto de um hospital público de referência do município de Picos - PI.

A população foi composta por todas as crianças nascidas vivas e por suas respectivas mães, no período de janeiro a dezembro de 2015, no referido hospital. A amostra foi censitária, os participantes foram selecionados de forma consecutiva, à medida que foram nascendo e preenchiam os critérios de elegibilidade, totalizando 546 nascidos vivos.

Os critérios de inclusão no estudo foram criança nascida viva, no período da coleta; criança cujo responsável aceitou participar da pesquisa e assinou o Termo de Consentimento Livre e Esclarecido. Foram considerados critérios de exclusão: recém-nascidos com baixa vitalidade ao nascer que impossibilite a permanência em alojamento conjunto; óbito fetal ou neonatal precoce; óbito materno; mãe com sorologia positiva para HIV no pré-natal registrada em prontuário.

Os dados foram coletados no período de janeiro a dezembro de 2015 , por meio de formulário adaptado de outros estudos, preenchido com a mãe ainda na maternidade do hospital ${ }^{9,10}$.

As variáveis utilizadas no estudo foram agrupadas em obstétricas, antropométricas dos recém-nascidos, tipos de AM, causas do não aleitamento e fatores de risco ao AME. Os dados obstétricos envolveram realização do pré-natal, quantidade de consultas realizadas, orientações sobre AM, profissional responsável pelas orientações, inspeção da mama, etilismo e tabagismo materno e tipo de parto.

Os dados antropométricos dos recém-nascidos abordaram peso, comprimento e perímetro cefálico. $\mathrm{O}$ peso (em gramas) foi classificado em baixo peso $(<$ $2.500)$, peso insuficiente (2.500-2.999), peso adequado (3.000-3.999) e excesso de peso $(>4.000) .{ }^{11}$ Para aferição do peso foi utilizada balança pediátrica mecânica da marca Welmy, devidamente calibrada, com a criança despida no momento da aferição.

O comprimento (em centímetros) foi classificado como menor que o esperado $(\leq 45)$, adequado (46 - 54) e maior que o esperado $(\geq 55) \cdot{ }^{11}$ A mensuração do comprimento foi realizada utilizando estadiômetro infantil de madeira, com a criança em decúbito dorsal sobre superfície plana e firme, mantendo joelhos em extensão e calcâneo apoiado, pé mantido em $90^{\circ}$. Para o perímetro cefálico (em centímetros) fez-se uso de fita métrica inelástica e flexível, como marcadores, utilizou-se a região frontal, occipital e linha acima da inserção da orelha, classificado como abaixo do esperado $(\leq 32)$ e normal $(>32)^{12}$.

Classificaram-se os tipos de aleitamento materno em AME, aleitamento predominante, aleitamento misto e aleitamento artificial ${ }^{13}$. As causas do não aleitamento foram categorizadas em criança não queria ???, leite insuficiente, problema no seio, criança doente, dificuldade na pega ou a criança estava dormindo. Foram considerados fatores de risco ao AME: uso de chupeta, mamadeira e problemas na mama.

Os dados foram organizados em tabelas e anali- 
sados com base em frequências absolutas e relativas. Para a realização do seguidos?? todos os princípios éticos contidos na resolução 466/12 que rege pesquisas envolvendo seres humanos ${ }^{14}$. O projeto foi aprovado pelo Comitê de Ética e Pesquisa da Universidade Federal do Piauí com parecer $\mathrm{n}^{0} 1.144 .279$. As mães que aceitaram participar da pesquisa assinaram o Termo de Consentimento Livre e Esclarecido (para mães com 18 anos de idade ou mais). Para as mães menores de 18 anos, foi solicitada a autorização do responsável legal, o qual assinou o Termo de Consentimento Livre e Esclarecido e a mãe o Termo de Assentimento Livre e Esclarecido.

\section{RESULTADOS}

Os resultados a seguir tratam do perfil obstétrico das mães, dos dados do nascimento dos recém-nascidos, do tipo de AM, das causas do não aleitamento e fatores de risco associados ao AME.

Das mães pesquisadas, 97,6\% realizaram consultas de pré-natal, sendo que $47 \%$ realizaram entre sete e nove consultas. Do total de mães, $67 \%$ receberam orientações sobre AM, destas 56,2\% foram orientadas por enfermeiros, no entanto, 30,2\% não receberam nenhuma orientação durante o pré-natal. Apenas 38,6\% tiveram sua mama examinada nesse período.

No que se refere ao alcoolismo materno, 7,9\% relataram ter feito uso de álcool durante a gestação. Quanto ao uso do cigarro, 7,1\% disseram ter feito uso antes da gravidez e 4,9\% durante a gravidez. Em relação ao tipo de parto, houve prevalência da cesariana $(75,3 \%)$. Estes dados encontram-se detalhados na Tabela 1.

Tabela 1. Distribuição das nutrizes por dados obstétricos. Picos, 2016

\begin{tabular}{lll} 
& & (Continua) \\
\hline Variáveis & $\mathbf{N = 5 4 6}$ & $\%$ \\
\hline Pré-Natal & & \\
Sim & 533 & 97,6 \\
Não & 11 & 2,0 \\
Não informada & 2 & 0,4 \\
\hline Quantidade de consultas de pré-natal & & \\
\hline $1-3$ & 30 & 5,5 \\
$4-6$ & 161 & 29,7
\end{tabular}

(Conclusão)

\begin{tabular}{lll}
\hline Variáveis & $\mathbf{N = 5 4 6}$ & \% \\
\hline $7-9$ & 255 & 47,0 \\
10 ou mais & 96 & 17,7 \\
\hline Orientações sobre AM & & \\
\hline Sim & 366 & 67,0 \\
Não & 167 & 30,6 \\
Não fez PN & 12 & 2,2 \\
Não informada & 1 & 0,2 \\
\hline
\end{tabular}

Profissional responsável pela orientação

\begin{tabular}{lll} 
Enfermeiro & 307 & 56,2 \\
Não recebeu orientação & 165 & 30,2 \\
Médico & 39 & 7,1 \\
Outros & 32 & 6,0 \\
Médico, enfermeiro e ACS & 2 & 0,4 \\
Não sabe & 1 & 0,2 \\
\hline
\end{tabular}

\section{Mama examinada}

\begin{tabular}{lll} 
Sim & 211 & 38,6 \\
Não & 321 & 58,8 \\
Não fez PN & 7 & 1,3 \\
Não sabe & 1 & 0,2 \\
Não informada & 6 & 1,1 \\
\hline
\end{tabular}

\section{Alcoolismo materno}

\begin{tabular}{lll} 
Sim & 43 & 7,9 \\
Não & 489 & 89,6 \\
Não informada & 14 & 2,6 \\
\hline
\end{tabular}

\begin{tabular}{lll}
\hline Tabagismo materno & & \\
\hline Antes da gravidez & 39 & 7,1 \\
Durante a gravidez & 27 & 4,9 \\
\hline Tipo de parto & & \\
\hline Cesárea & 411 & 75,3 \\
Normal & 131 & 24,0 \\
Fórceps & 2 & 0,4 \\
Não sabe & 2 & 0,4 \\
\hline
\end{tabular}

Fonte: Dados da pesquisa.

Os recém-nascidos avaliados apresentaram, ao nascimento, 69,6\% peso adequado, 91,6\% comprimento adequado, 95,4\% valores normais de perímetro cefálico. Quanto ao sexo houve predominância do sexo feminino, conforme exposto na Tabela 2. 
Tabela 2. Distribuição dos recém-nascidos por dados do nascimento. Picos, 2016

\begin{tabular}{lll}
\hline Variáveis & $\mathrm{N}=546$ & $\%$ \\
\hline Peso $(\mathrm{g})$ & & \\
\hline Baixo $(<2.500)$ & 25 & 4,6 \\
Peso insuficiente $(2.500-2.999)$ & 119 & 21,8 \\
Peso adequado $(3.000-3.999)$ & 380 & 69,6 \\
Excesso de peso $(>4.000)$ & 22 & 4 \\
\hline Comprimento (cm) & & \\
\hline Menor que o esperado $(\leq 45)$ & 37 & 6,8 \\
Adequado $(46-54)$ & 500 & 91,6 \\
Maior que o esperado $(\geq 55)$ & 4 & 7 \\
Não informado & 5 & 9 \\
\hline Perímetro cefálico $(\mathrm{cm})$ & & \\
\hline Abaixo do esperado $(\leq 32)$ & 18 & 3,3 \\
Normal $(>32)$ & 521 & 95,4 \\
Não informado & 7 & 1,3 \\
\hline Sexo & 278,9 \\
\hline Feminino & & \\
Masculino & & \\
\hline
\end{tabular}

Fonte: Dados da pesquisa.

A maioria dos recém-nascidos $(92,7 \%)$ encontra-se em AME. Uma pequena proporção destes recebeu água, chá ou outro nutriente, estando $2,9 \%$ em AM predominante, $1,1 \%$ em AM misto e 1,1\% em aleitamento artificial, conforme apresentado na Tabela 3 .

Tabela 3. Distribuição da amostra quanto ao tipo de aleitamento. Picos, 2016

\begin{tabular}{lll}
\hline Variáveis & Ao nascer & \\
\hline & $\mathrm{N}=546$ & $\%$ \\
\hline AME & 506 & 92,7 \\
Predominante & 16 & 2,9 \\
Misto & 6 & 1,1 \\
Artificial & 6 & 1,1 \\
Não informada & 12 & 2,2 \\
\hline
\end{tabular}

Fonte: Dados da pesquisa.
No que diz respeito às causas do não aleitamento, a Tabela 4 mostra que 3,1\% das mães consideram seu leite insuficiente para saciar a fome do recém-nascido e $3,7 \%$ relataram que a criança não queria mamar, e, portanto, foi introduzida uma alimentação, a fim de complementar o AM. Em relação ao uso de chupeta e mamadeira ao nascer, constatou-se que $20,7 \%$ das crianças faziam uso de chupeta e $4,4 \%$ de mamadeira. Quanto aos problemas na mama, as mulheres relataram mamilos dolorosos, mamilos planos e invertidos, fissura mamilar, ingurgitamento dos seios, ductos obstruídos e mastite.

Tabela 4. Motivos alegados para a não amamentação e fatores de risco ao AME. Picos, 2016

\begin{tabular}{|c|c|c|}
\hline Variáveis & $N=546$ & $\%$ \\
\hline \multicolumn{3}{|l|}{ Motivos para o não aleitamento } \\
\hline Criança não queria & 20 & 3,7 \\
\hline Leite insuficiente & 17 & 3,1 \\
\hline Problema no seio & 6 & 1,1 \\
\hline Não sabe & 4 & 0,8 \\
\hline Criança doente & 3 & 0,5 \\
\hline Dificuldade na pega & 3 & 0,6 \\
\hline Criança dormindo & 2 & 0,5 \\
\hline \multicolumn{3}{|l|}{ Uso de chupeta } \\
\hline Sim & 113 & 20,7 \\
\hline Não & 432 & 79,1 \\
\hline Não informada & 1 & 0,2 \\
\hline \multicolumn{3}{|l|}{ Uso de mamadeira } \\
\hline Sim & 24 & 4,4 \\
\hline Não & 519 & 95,1 \\
\hline Não informada & 3 & 5,0 \\
\hline \multicolumn{3}{|l|}{ Problema na mama } \\
\hline Mamilos dolorosos & 19 & 3,5 \\
\hline Mamilos planos ou invertidos & 13 & 2,4 \\
\hline Fissura mamilar & 17 & 3,1 \\
\hline Ingurgitamento dos seios & 4 & 0,8 \\
\hline Ductos obstruídos e mastite & 3 & 0,6 \\
\hline Nenhum & 487 & 89,2 \\
\hline Não informada & 3 & 5,0 \\
\hline
\end{tabular}

Fonte: Dados da pesquisa. 


\section{DISCUSSÃO}

A manutenção de práticas alimentares adequadas na infância é essencial para a garantia da sobrevida e saúde das crianças, incluindo condições ótimas de estado nutricional, crescimento e desenvolvimento, sendo o AM uma das mais importantes práticas promotoras de saúde infantil, tendo reflexos positivos durante toda a vida.

Desta forma, é necessário conhecer a distribuição das nutrizes por dados obstétricos, uma vez que a gravidez pode influenciar no processo de não aleitamento, aleitamento não exclusivo e/ou desmame precoce. Os resultados do estudo mostram que $97,6 \%$ das mães pesquisadas realizaram o pré-natal e que $67 \%$ receberam orientações sobre AM, corroborando com estudo de Barbosa $^{3}$ que constatou que $99,7 \%$ do total das mães realizaram o pré-natal e com estudo de Carvalho et al. ${ }^{15}$ que evidenciou que $76,6 \%$ das mães receberam orientações sobre AM durante o pré-natal.

O pré-natal é um período oportuno para que o profissional enfermeiro oriente as gestantes quanto à importância do AM e as dificuldades que poderão enfrentar durante o processo de lactação $0^{4}$. As abordagens acerca do AM durante o pré-natal são decisivas para a prática de amamentar e sua duração, visto que as mulheres devem construir nesse período a compreensão que influenciará no sucesso da amamentação $0^{3}$. A educação e o preparo das mulheres possuem uma boa atuação no sentido de promover, proteger e apoiar a amamentação exclusiva até os seis meses de vida da criança ${ }^{16}$.

Ao analisar a amostra quanto ao etilismo e tabagismo, observa-se que um pequeno percentual de mães fez uso de álcool ou tabaco no período gestacional. Mães que relataram fazer uso do álcool e do tabaco tem menor duração de aleitamento materno em relação as outras mães que não fizeram uso dos mesmos ${ }^{17}$.

Com relação ao tipo de parto predominou a cesariana, corroborando com estudos de Gonçalves ${ }^{18}$, Paula Júnior et al. ${ }^{19}$ e de Sampaio, Bousquat e Barros ${ }^{20}$ que encontraram $80 \%, 55,17 \%$ e 51,4\% de partos cesáreos, respectivamente. Estes últimos observaram que nenhuma paciente submetida ao parto cesariano teve oportunidade de realizar o quarto passo dos dez recomendados pela Iniciativa Hospital Amigo da Criança, que consiste em colocar os bebês em contato pele a pele com suas mães imediatamente após o parto por, no mínimo, 01 hora, encorajando-as a reconhecer quando seus bebês estão prontos para serem amamentados da maneira preconizada. Em contraponto, estudo que avaliou a duração do aleitamento materno e fatores associados entre $1960 \mathrm{e}$ 2000 não encontrou associação entre o tipo de parto e o risco de interrupção do aleitamento materno ${ }^{21}$.

Quanto à distribuição dos recém-nascidos por dados do nascimento, predominou peso adequado, comprimento adequado, perímetro cefálico normal e sexo feminino, semelhante à pesquisa de Figueredo, Mattar e Abrão ${ }^{22}$ que encontrou média do peso ao nascer de 3.345 gramas. Quando a criança nasce com baixo peso, gera tensão maior nas mães associada à insegurança e preocupação de como fazer a criança obter o peso adequado, exigindo maior habilidade e preparo por parte da mãe ${ }^{17}$.

$\mathrm{Na}$ distribuição quanto ao tipo de aleitamento ao nascer, predominou o AME, corroborando com estudo de Borges et al. ${ }^{23}$ no qual $64 \%$ das mulheres entrevistadas ofereceram exclusivamente o leite humano aos seus bebês. A amamentação exclusiva traz vantagens não só para o bebê como também para a mãe, como perda do peso ganho durante a gravidez, eficaz método anticoncepcional e redução do risco de desenvolvimento de câncer de mama.

Amamentar não é um ato totalmente instintivo no ser humano, geralmente é um processo que deve ser aprendido para se ter sucesso, levando em consideração que as nutrizes necessitam de esforço e apoio constantes, especialmente as primíparas, que ao se depararem pela primeira vez com o aleitamento materno, têm como referencial maior o seio familiar, as amizades e a vizinhança onde está inserida ${ }^{18}$.

No que se refere às causas do não aleitamento o presente estudo revela que a maioria das mães relatou que o seu leite era insuficiente para saciar a fome do bebê e/ou que a crianças não queriam. Em estudo realizado por Araújo et al. ${ }^{24} 26,5 \%$ da amostra relataram não ter leite suficiente e 5,9\% relataram problemas nas mamas.

A mãe interpreta o choro da criança como ausência de saciedade da fome, e espera-se que a criança durma após mamar, então para a mãe o seu leite é insuficiente e de baixa qualidade para atender as demandas nutricio- 
nais do seu filho. Todavia, não existe "leite fraco", todo leite materno é forte e bom, a cor do leite pode variar, mas ele nunca é fraco, a maioria das mulheres tem condições biológicas de produzir leite ${ }^{13}$.

A distribuição do estudo quanto aos fatores de risco ao AME focou no uso de chupetas e mamadeiras, o uso desses fatores de risco pode contribuir para alterar a sucção do bebê, pela quantidade de líquido da mama da mãe ser menor que o volume da mamadeira, exigindo, portanto, menor esforço ${ }^{25}$. A Organização Mundial da Saúde recomenda a não utilização de bicos artificiais ou chupetas em crianças que estão sendo amamentadas por contribuírem para o encerramento precoce de amamentação $0^{26}$.

Estudo que avaliou 319 mães atendidas na rede pública de saúde de Palmas-TO mostrou que 77\% destas não ofereciam mamadeiras às crianças, entre as que utilizavam mamadeiras estavam aquelas que ofertavam fórmulas lácteas artificiais, outros complementos líquidos e leite humano ordenhado. ${ }^{23} \mathrm{Em}$ contrapartida, estudo de Araújo et al. ${ }^{24}$ atestou que $61,1 \%$ das mães já haviam utilizado chupeta ou mamadeira, ou pelo menos um destes em seus filhos. Crianças que não fazem uso de chupeta apresentam $89 \%$ maior chance de $\mathrm{AME}^{27}$. As crianças que usam chupeta mamam com menos frequência, o que pode prejudicar a produção do leite materno, levando ao desmame precoce, causando uma "confusão de sucção" ou "confusão de bicos" causada pela facilidade de sugar na chupeta ${ }^{4}$. As experiências orais precoces que favorecem o uso de mecanismos de sucção diferentes dos utilizados no aleitamento materno, podem causar alteração na pega e, consequentemente, fracasso no aleitamento ${ }^{26}$.

Tendo em vista as consequências negativas advindas das práticas de não aleitamento e desmame precoce, são necessárias intervenções específicas e eficazes por parte dos profissionais de saúde, especialmente o enfermeiro, com o escopo de que as mães se sintam assistidas em suas dúvidas e dificuldades, garantindo o cuidado durante a gestação e na assistência à nutriz no pós-parto.

\section{CONCLUSÃO}

Conclui-se que os objetivos da pesquisa foram alcançados, pois foi possível investigar a prevalência do aleitamento materno exclusivo ao nascer e seus fatores de risco em crianças.

A principal limitação do estudo foi o viés de memória das mães pesquisadas quanto às informações fornecidas, de modo que podem não ter se lembrado de mencionar alguma orientação recebida durante a gestação, devido, principalmente, a emoção por conhecer seu bebê, bem como por possível estresse durante e após o parto e a primeira mamada da criança.

É importante que as mães sintam que os profissionais de saúde estão aptos para auxiliá-la, para se sentirem apoiadas e confiantes, sendo necessário realizar intervenções de promoção ao aleitamento materno e prevenção do desmame precoce por meio de atividades educativas que orientem essas mães e também a família envolvida nesse processo de amamentação quanto às dúvidas, aos mitos e às crenças sobre o ato de amamentar, a fim de que elas se sintam assistidas em suas dúvidas e dificuldades.

\section{REFERÊNCIAS}

1. Moura LP, Oliveira JM, Noronha DD, Torres JDRV, Oliveira KCF, Teles MAB. Percepção de mães cadastradas em uma estratégia saúde da família sobre aleitamento materno exclusivo. Rev enferm UFPE online. 2017;11(3):1403-9.

2. Rollins NC, Bhandari N, Hajeebhoy N, Horton S, Lutter CK, Martines JC, et al. Why invest, and what it will take to improve breastfeeding practices?. The Lancet. 2016;387(10017):491-504.

3. Barbosa LN, Santos NC, Morais MAM, Rizzardi SD, Corrêa EC. Prevalência de práticas educativas acerca do aleitamento materno exclusivo (AME) em Cuiabá - MT. Esc Anna Nery. 2015;19(11):147-53.

4. Rufino VSP. Investigação das causas do desmame precoce em mães picoenses. 2014 [Monografia]. Picos: Universidade Federal do Piauí; 2014. 47 p.

5. Tenório MCS, Dutra TA, Lima AA, Monteiro JRS, Oliveira ACM. Prevalência e fatores associados ao 
início tardio do aleitamento materno em recém-nascidos prematuros nascidos em maternidade de referência de alto risco de Maceió, Alagoas. Gep News. 2018;2(2):63-9.

6. Ministério da Saúde (BR). II Pesquisa de Prevalência de Aleitamento Materno nas Capitais Brasileiras e Distrito Federal. Brasília (DF): Ministério da Saúde; 2009.

7. UNICEF. State of the World's Children. Nova York: UNICEF; 2013.

8. Paim JSL, Boiani MB, Freitas TS. Fatores associados a pratica e a duração do aleitamento materno no Brasil contemporâneo. Investigação. 2018;7(3):66-74.

9. Boccolini CS, Carvalho ML, Oliveira MIC, Vasconcelos AGG. Fatores associados à amamentação na primeira hora de vida. Rev Saúde Pública. 2011;45(1):69-78.

10. Caminha MFC, Batista Filho M, Serva VB, Arruda IKG, Figueiroa JN, Lira PIC. Tendências temporais e fatores associados à duração do aleitamento materno em Pernambuco. Rev Saúde Pública. 2010;44(2):240-8.

11. Souza AG. Enfermagem Neonatal: Cuidado integral ao recém-nascido. São Paulo: Martinari; 2011.

12. Ministério da Saúde (BR). Secretaria de Vigilância em Saúde. Protocolo de vigilância e resposta à ocorrência de microcefalia e/ou alterações do sistema nervoso central (SNC). 2. ed. Brasília (DF): Ministério da Saúde; 2016.

13. Ministério da Saúde (BR). Secretaria de Atenção à Saúde. Departamento de Atenção Básica. Saúde da criança: nutrição infantil: aleitamento materno e alimentação complementar. Brasília (DF): Ministério da Saúde; 2015.

14. Ministério da Saúde (BR). Conselho Nacional de Saúde. Resolução 466/12. Brasília (DF): Ministério da Saúde; 2012.

15. Carvalho JLS, Cirino IP, Lima LHO, Sousa AF, Carvalho MF, Oliveira EAR. Conhecimento das mães sobre aleitamento materno exclusivo e alimentação complementar. Saúde em Redes. 2016;2(4):383-92.

16. Macedo MDS, Torquato IMB, Trigueiro JVS, Albuquerque AM, Pinto MB, Nogueira MF. Aleitamento materno: identificando a prática, benefícios e os fatores de risco para o desmame precoce. Rev enferm UFPE online. 2015;9(1):414-23.
17. Victoria CG, Bahl R, Barros AJD, França GVA, Horton S, Krasevec J, et al. Breastfeeding in the 21st century: epidemiology, mechanisms, and lifelong effect. The Lancet. 2016; 387(10017):475-90.

18. Gonçalves LS. Conhecimento de mães adolescentes assistidas na atenção primária sobre o desmame precoce [Monografia]. Picos: Universidade Federal do Piauí; 2013. 50 p.

19. Paula Júnior JD, Lucas ES, Cunha LMC, Machado MGM, Pedrosa RL. Perfil da mortalidade neonatal no município de Ubá/MG, Brasil (2008-2010). Rev Bras Pesq Saúde. 2016;18(3):24-31.

20. Sampaio ARR, Bousquat A, Barros C. Contato pele a pele ao nascer: um desafio para a promoção do aleitamento materno em maternidade pública no Nordeste brasileiro com o título de Hospital Amigo da Criança. Epidemiol Serv Saude. 2016:25(2):281-90.

21. Oliveira DS, Boccolini CS, Faersteina E, Verly-jr E. Duração do aleitamento materno e fatores associados entre 1960 e 2000. J Pediatr. 2017;93(2):130-35.

22. Figueredo SF, Mattar MJG, Abrão ACFV. Hospital Amigo da Criança: prevalência de aleitamento materno exclusivo aos seis meses e fatores intervenientes. RevEscEnferm. 2013;47(6):1291-97.

23. Borges NR, Sousa DKS, Pereira RJ, Castro JGD. Caracterização e prevalência do aleitamento materno em uma população atendida na rede pública de saúde de Palmas/TO, Brasil. Rev Bras Pesq Saúde. 2016;18(4):30-6.

24. Araújo JP, Almeida JLS, Souto CMRM, Oliveira AEA, Sudério MARP. Desmame precoce e suas causas: experiência na atenção básica de Campina Grande-PB. Revista da Universidade Vale do Rio Verde. 2013;11(2):146-55.

25. Barbosa JAG, Santos FPC, Silva PMC. Fatores associados a baixa adesão ao aleitamento materno exclusivo e ao desmame precoce. Rev Tecer. 2013;6(11):15465 .

26. Batista CLC, Ribeiro VS, Nascimento MDS. Influência do uso de chupetas e mamadeiras na prática do aleitamento materno. J Health BiolSci. 2017;5(2):184-91.

27. Ducci AL, Vannuchi MTO, Tacla MTGM, Souza SNDH, Reis TB. Prevalência e fatores associados ao aleitamento materno exclusive em menores de seis meses 
no município de Rolândia- PR. Reme. 2013;17(2):145-

53.

Recebido em: 09/08/2018

Aceito em: 04/09/2018 